\title{
A Simulation-based Approach to Stochastic Dynamic Programming
}

\author{
Nicholas G. Polson \\ Morten Sorensen \\ Booth School of Business* \\ GSB, Columbia \\ First Draft: November 2007 \\ This Draft: November 2010
}

\begin{abstract}
In this paper we develop a simulation-based approach to stochastic dynamic programming. To solve the Bellman equation we construct Monte Carlo estimates of Q-values. Our method is scalable to high dimensions and works in both continuous and discrete state and decision spaces whilst avoiding discretization errors that plague traditional methods. We provide a geometric convergence rate. We illustrate our methodology with a dynamic stochastic investment problem.
\end{abstract}

Keywords: Q-Learning, Bellman, Dynamic Programming, Sequential Monte Carlo, Stochastic Simulation, Simulated Annealing.

*Address for correspondence: University of Chicago, 5807 South Woodlawn Avenue, Chicago, IL 60637, U.S.A. E-mail: ngp@chicagobooth.edu. We thank Seung Min Yae for invaluable research assistance. 


\section{Introduction}

We develop a simulation-based strategy for solving Bellman's dynamic programming problem. Specifically, we develop a sequential Monte Carlo algorithm to find the associated $Q$-values, which, in turn, define the value and optimal policy functions. Gor Monte Carlo approaches to solving the Bellman equation, via backwards induction, see Brockwell and Kadane (2003), Virto et al. (2003), Rust (1997), Mueller (1999), Mueller et al. (2004), Amzal et al. (2006) and Brown et al. (2010).

Our approach provides an alternative to the $Q$-Learning algorithm of Watkins (1989), which has been widely applied across fields. In statistics and machine learning, dynamic programming is central to optimal control and design of experiments (Whittle, 1969, Gittins, 1979, Bertsekas, 1994), optimization over time (Whittle, 1983), neuro-dynamic programming (Bertsekas and Tsitsiklis, 1996) and reinforcement learning (Lovejoy, 1991, Puterman, 1994, Sutton and Barto, 1998). In economics and finance, it is central to describing agents' dynamic decision making given uncertainty over asset prices and other economic variables (Judd, 1999, Ljungqvist and Sargent, 2004).

Our method draws on insights from solving inverse problems using simulated annealing (Kirkpatrick et al., 1984, Aarts and Korst, 1997, Forsythe and Leibler, 1950, Cutokswy, 1951, Curtiss, 1953, Hammersley and Handscomb, 1964, Halton, 1970, Polson and Roberts, 1993 and Barto and Duff, 1994). We show how sequential Monte Carlo methods overcome the curse of dimensionality. Compared to traditional approachers, our method can be more scalable, and applies to both discrete and continuous problems while avoiding discretization errors.

We provide a geometric convergence result for our method. Sutton (1999) remarks that developing convergence results for reinforcement learning algorithms is one of the most important open theoretical questions in this literature. To illustrate the application of our 
methodology, we solve a simple dynamic stochastic investment problem.

The two primary approaches for solving dynamic programming problems are iterating on either the value function (Bellman, 1956) or the policy function (Howard, 1960). Iterating on the policy function has a second-order geometric convergence rate (see, for example, Whittle and Komarov, 1987). However, value and policy iteration require matrix inversion which is $O\left(N^{3}\right)$ and are thus not scalable. Our sequential Monte Carlo strategy provides scalability by constructing a random operator for iterations that allows us to evaluate in $O(N)$ the $Q$-values at any point in both the state and decision spaces.

There are also deterministic approaches based on parametric weighted combinations of basis functions to approximate the value or policy function. Bellman et al. (1963) propose polynomial approximations, and Dantzig et al. (1974), Whitt (1978) and Judd and Solnick (1994) discuss the advantages of spline-based methods. Tauchen (1990) and Tauchen and Hussey (1991) propose a quadrature-based method.

The paper is outlined as follows. Section 2 describes our random Monte Carlo operator for computing $Q$-values and the construction of the algorithm. Section 3 proves geometric convergence and discusses the properties of the algorithm. Section 4 illustrates our methodology in a dynamic stochastic investment problem. Section 5 provides conclusions.

\section{Bellman Optimality via Simulation}

Consider a standard dynamic programming problem. An agent has intertemporal preferences represented by the utility function $U=\sum_{t} \beta^{t} u(s(t), d(t))$, where $\beta$ is the agent's discount rate, $s(t)$ is a sequence of states, and $d(t)$ is a sequence of decisions made by the agent. The agent's per-period utility is $u(s, d)$, where $s$ is the current state, and $d$ is the current decision. Next period's state, $s^{\prime}$, depends on the current state and decision with known transition probability $p\left(s^{\prime} \mid s, d\right)$. The aim is to determine the utility-maximizing decision, 
$d^{\star}(s)$, taking into account the decision's effects on future states and decisions.

The principle of optimality states that $d^{\star}(s)$ is the solution to the Bellman equation (Bellman, 1956) with value function $V(s)$ defined by

$$
V(s)=\max _{d^{\star}}\left(u\left(s, d^{\star}\right)+\beta \int V\left(s^{\prime}\right) p\left(s^{\prime} \mid s, d^{\star}\right) d s^{\prime}\right) .
$$

In contrast, $Q$-values are defined as the total expected utility gained from choosing the current action, $d$, and then following the optimal decision rule afterwards. By definition,

$$
\begin{aligned}
Q(s, d) & =u(s, d)+\beta \int V\left(s^{\prime}\right) p\left(s^{\prime} \mid s, d\right) d s^{\prime} \\
V(s) & =\max _{d^{\star}} Q\left(s, d^{\star}\right) \\
d^{\star}(s) & =\arg \max _{d^{\star}} Q\left(s, d^{\star}\right),
\end{aligned}
$$

and the Bellman equation can be restated in terms of Q-values as

$$
Q(s, d)=u(s, d)+\beta \int \max _{d^{\star}} Q\left(s^{\prime}, d^{\star}\right) p\left(s^{\prime} \mid s, d\right) d s^{\prime} .
$$

\subsection{Q-Learning: Stochastic Approximation}

Before we describe our sequential Monte Carlo approach, we outline the popular $Q$-Learning (Watkins, 1989) stochastic approximation algorithm (Robbins and Munro, 1951), which allows us to introduce some useful notation. Related approaches include temporal difference (TD) learning (Sutton, 1988) and Monte Carlo evolutionary strategies (Sutton, 1999). The use of Monte Carlo methods is paramount in high dimensions as it provides a way to carry out the Bellman fixed-point iteration by approximating expectations through random sampling.

A sequence of functions $Q^{(g)}$ that converges to the $Q$-values can be computed by itera- 
tively evaluating

$$
Q^{(g+1)}(s, d)=u(s, d)+\beta \int \max _{d^{\star}} Q^{(g)}\left(s^{\prime}, d^{\star}\right) p\left(s^{\prime} \mid s, d\right) d s^{\prime}
$$

starting at $Q^{(0)}(s, d)=0$. Watkins (1989) presents a stochastic version of this approach. Bellman's equation for $Q$-values can be written as

$$
E_{s^{\prime} \mid s, d}\left(Q(s, d)-\beta \max _{d^{\star}} Q\left(s^{\prime}, d^{\star}\right)\right)=u(s, d),
$$

and this equation can be solved for $Q$ through stochastic approximation. This simulationbased approach proceeds by simulating states $s^{\prime} \sim p\left(s^{\prime} \mid s, d\right)$ and computing the sequence

$$
Q^{(g+1)}\left(s^{\prime}, d\right)=Q^{(g)}\left(s^{\prime}, d\right)+a^{(g)}\left(u\left(s^{\prime}, d\right)-Y^{(g)}\left(s^{\prime(g)}, Q^{(g)}\right)\right)
$$

where $Y^{(g)}\left(s^{\prime(g)}, Q^{(g)}\right)=Q^{(g)}\left(s^{\prime}, d\right)-\beta \max _{d^{\star}} Q\left(s^{\prime}, d^{\star}\right)$. The sequence $a^{(g)}$ is chosen to satisfy $\sum_{g=1}^{\infty} a^{(g)}=\infty$ and $\sum_{g=1}^{\infty}\left(a^{(g)}\right)^{2}<\infty$ for convergence of $Q^{(g)} \rightarrow Q$ (see Watkins and Dayan, 1992). To avoid the specification of a sequence of learning coefficients, Lagondakis et al. (2002) and Lagondakis and Parr (2003) propose a least-squares policy iteration algorithm.

A main caveat with this approach is that it is infeasible in continuous and high-dimensional settings as it requires an explicit discretization of the state and decision spaces to evaluate $\max _{d^{\star}} Q\left(s^{\prime}, d^{\star}\right)$. In contrast, we use sequential Monte Carlo, together with insights from simulated annealing, to calculate the $Q$-values. 


\subsection{Q-Values: Simulated Annealing}

To avoid direct computation of $\max _{d^{\star}} Q\left(s^{\prime}, d^{\star}\right)$, we use a simulated annealing random operator. Define the annealed transition distribution for decision $d^{\star}$ by

$$
p_{\lambda}^{Q}\left(d^{\star} \mid s^{\prime}\right) \sim \frac{e^{\lambda Q\left(s^{\prime}, d^{\star}\right)}}{\int_{d^{\star}} e^{\lambda Q\left(s^{\prime}, d^{\star}\right)} d \mu\left(d^{\star}\right)}
$$

for some mesaure $\mu\left(d^{\star}\right)$ to ensure integrability. For asymptotically large $\lambda$, we can compute $\max _{d^{\star}} Q\left(s^{\prime}, d^{\star}\right)$ via

$$
\int p_{\lambda}^{Q}\left(d^{\star} \mid s^{\prime}\right) Q\left(s^{\prime}, d^{\star}\right) d \mu\left(d^{\star}\right) \rightarrow \max _{d^{\star}} Q\left(s^{\prime}, d^{\star}\right)
$$

See Pincus (1968), Aarts and Korst (1988), Kirkpatrick (1983) and Robert and Casella (2006) for further discussion.

The annealed version of Bellman's equation for $Q$-values is then a nonlinear functional integral equation

$$
\begin{aligned}
Q(s, d) & =u(s, d)+\beta \iint p_{\lambda}^{Q}\left(d^{\star} \mid s^{\prime}\right) p\left(s^{\prime} \mid s, d\right) Q\left(s^{\prime}, d^{\star}\right) d \mu\left(s^{\prime}, d^{\star}\right) \\
& =u(s, d)+\beta \int P^{Q}\left(s^{\prime}, d^{\star} \mid s, d\right) Q\left(s^{\prime}, d^{\star}\right) d \mu\left(s^{\prime}, d^{\star}\right)
\end{aligned}
$$

with the joint transition probability from $(s, d)$ to $\left(s^{\prime}, d^{\star}\right)$ defined by

$$
P^{Q}\left(s^{\prime}, d^{\star} \mid s, d\right)=p_{\lambda}^{Q}\left(d^{\star} \mid s^{\prime}\right) p\left(s^{\prime} \mid s, d\right) .
$$

This reduces the problem of solving Bellman's equation to solving the nonlinear functional equation

$$
Q(s, d)=u(s, d)+\beta P^{Q}(s, d) Q,
$$


where, for any integrable functional $f\left(s^{\prime}, d^{\star}\right)$, we define

$$
P^{Q}(s, d) f=\int P^{Q}\left(s^{\prime}, d^{\star} \mid s, d\right) f\left(s^{\prime}, d^{\star}\right) d \mu\left(s^{\prime}, d^{\star}\right)
$$

This operator can be interpreted as follows. Arriving from state $s$ and making decision $d$, the transition dynamics $P^{Q}\left(s^{\prime}, d^{\star} \mid s, d\right)$ induces a distribution over future states and decisions. The operator takes the expectation of $Q$ under this induced distribution. The benefits of this formulation are three-fold: First, given an accurate approximation of $P^{Q}(s, d) Q$, we can evaluate $Q(s, d)$ for any $(s, d)$ pair. Second, in high-dimensional decision spaces, where maximums are difficult to compute directly, our formulation solves for the maximum in a probabilistic sense, using simulated annealing. Finally, viewing $d^{\star}$ as a stochastic variable allows us to formulate a joint transition kernel $P^{Q}\left(s^{\prime}, d^{\star} \mid s, d\right)$ for moving from the current state $(s, d)$ to $\left(s^{\prime}, d^{\star}\right)$.

\subsection{Constructing the Random Operator}

We now show how to approximate $P^{Q}(s, d) f$ using the random operator $\mathcal{P}^{Q}(s, d) f$. The random operator $\mathcal{P}^{Q}(s, d)$ will be computable for any $(s, d)$ pair, thus avoiding discretization of the original problem. To define $\mathcal{P}$, we generate a stochastic grid $\left(s_{i}^{\prime}, d_{i}^{\star}\right)_{i=1}^{M}$, which is updated at each iteration to maintain the statistical properties of the operator. Specifically, given $Q^{(g)}$, we approximate $P^{Q^{(g)}}(s, d) Q^{(g)}$ by drawing $\left(s_{i}^{\prime}, d_{i}^{\star}\right)_{i=1}^{M}$ from a suitably defined distribution, $p^{(g)}\left(s^{\prime}, d^{\star}\right)$, and evaluating

$$
\mathcal{P}^{Q^{(g)}}(s, d) Q^{(g)}=\frac{1}{M} \sum_{i=1}^{M} \frac{P^{Q^{(g)}}\left(s_{i}^{\prime}, d_{i}^{\star} \mid s, d\right)}{p^{(g)}\left(s_{i}^{\prime}, d_{i}^{\star}\right)} Q^{(g)}\left(s_{i}^{\prime}, d_{i}^{\star}\right)
$$


where, by definition,

$$
\begin{aligned}
P^{Q^{(g)}}\left(s^{\prime}, d^{\star} \mid s, d\right) & =p_{\lambda}^{Q^{(g)}}\left(d^{\star} \mid s^{\prime}\right) p\left(s^{\prime} \mid s, d\right) \\
p_{\lambda}^{Q^{(g)}}\left(d^{\star} \mid s^{\prime}\right) & \sim \frac{e^{\lambda Q^{(g)}\left(s^{\prime}, d^{\star}\right)}}{\int_{d^{\star}} e^{\lambda Q^{(g)}\left(s^{\prime}, d^{\star}\right)} d \mu\left(d^{\star}\right)}
\end{aligned}
$$

For $p^{(g)}\left(s^{\prime}, d^{\star}\right)$, it is natural to choose

$$
p^{(g)}\left(s^{\prime}, d^{\star}\right)=p_{\lambda}^{Q^{(g)}}\left(d^{\star} \mid s^{\prime}\right) p\left(s^{\prime}\right),
$$

where $p\left(s^{\prime}\right)$ is the uniform distribution over the state space.

This choice yields a convenient expression for $\mathcal{P}^{Q^{(g)}}(s, d) Q^{(g)}$ namely

$$
\mathcal{P}^{Q^{(g)}}(s, d) Q^{(g)}=\frac{1}{M} \sum_{i=1}^{M} \frac{p\left(s_{i}^{\prime} \mid s, d\right)}{p\left(s_{i}^{\prime}\right)} Q^{(g)}\left(s_{i}^{\prime}, d_{i}^{\star}\right)
$$

This random operator can be evaluated at any $(s, d)$ pair and forms the core of our algorithm.

The random operator $\mathcal{P}^{(g)}$ has the usual statistical properties

$$
\begin{aligned}
E\left(\mathcal{P}^{(g)}(s, d) Q^{(g)}\right) & =P^{Q^{(g)}}(s, d) Q^{(g)} \\
\operatorname{Var}\left(\mathcal{P}^{(g)}(s, d) Q^{(g)}\right) & =\frac{C(s, d)}{M}
\end{aligned}
$$

where

$$
C(s, d)=\operatorname{Var}_{\left(s^{\prime}, d^{\star}\right)}\left(\frac{p\left(s^{\prime} \mid s, d\right)}{p\left(s^{\prime}\right)} Q^{(g)}\left(s^{\prime}, d^{\star}\right)\right)
$$

We assume that these moments are finite for all $(s, d)$ points. Hence, the Monte Carlo error in using $\mathcal{P}^{(g)}$ to approximate $P^{(g)}$ is $O\left(M^{-1}\right)$.

With a discrete decision space, constructing the random grid $\left(s_{i}^{\prime}, d_{i}^{\star}\right)_{i=1}^{M}$ requires drawing $p^{Q^{(g)}}\left(d^{\star} \mid s^{\prime}\right)$ as a multinomial draw, an $O(N)$ operation, for each $s_{i}^{\prime}$. For a continuous decision 
space, we can simulate candidate values $d_{i}^{\star}$ using a multinomial draw with the appropriate weights, or via slice sampling.

\subsection{Algorithm}

As described, the operator reduces the problem of solving Bellman's equation to a nonlinear functional equation of the form $Q(s, d)=u(s, d)+\beta P^{Q}(s, d) Q$. In discrete and continuous cases, this is a matrix or functional equation, respectively. Our framework has the additional stochastic component of approximating $P^{Q}(s, d) Q$ using the random operator $\mathcal{P}^{(g)}(s, d) Q^{(g)}$, discussed previously. We start from the iteration

$$
Q^{(g+1)}(s, d)=u(s, d)+\beta P^{Q^{(g)}}(s, d) Q^{(g)}
$$

Replacing the operator $P^{Q}$ by the random operator $\mathcal{P}^{Q}$, leads to the associated random operator equation

$$
Q_{M}^{(g+1)}(s, d)=u(s, d)+\beta \mathcal{P}_{M}^{(g)}(s, d) Q_{M}^{(g)}
$$

If we define the residual by $Q(s, d)-Q^{(g)}(s, d)$ and estimate it by $\hat{Q}^{(g)}(s, d)$, we can alternatively formulate the iterations as

$$
Q^{(g+1)}(s, d)=Q^{(g)}(s, d)+\hat{Q}^{(g)}(s, d)
$$

leading to a sequence of $Q$-values, $Q^{(g)}(s, d)$, defined by the recursion

$$
\begin{gathered}
u^{(g)}(s, d)=u(s, d)-Q^{(g)}(s, d)+\beta \mathcal{P}^{Q^{(g)}}(s, d) Q^{(g)} \\
Q^{(g+1)}(s, d)_{M}=Q^{(g)}(s, d)+u^{(g)}(s, d)+\beta \mathcal{P}^{Q^{(g)}}(s, d) u^{(g)}
\end{gathered}
$$

We now derive convergence properties for these iterative algorithms. 


\section{Geometric Convergence Properties}

We prove first-order geometric convergence for our algorithm by extending ideas from the linear case (Curtiss, 1954), to the nonlinear case. For related convergence results for random mappings, see Diaconis and Freedman (1999) and Halton (2006). For convergence properties of TD and Q-Learning, see Dayan (1992) and Watkins and Dayan (1992), respectively.

Jakkola et al. (1994), Singh et al. (1998), Tesuro and Galperin (1996), Tsitsikis (1994, 2002) and Kearns and Singh $(1998,1999)$ provide a number of convergence results for related Q-learning algorithms.

\subsection{Properties of the Annealing Function}

It is useful to develop some properties of the annealing function. For $q \in R^{I}$,

$$
f_{i}(q)=\frac{\exp \left(\lambda q_{i}\right)}{\sum_{j} \exp \left(\lambda q_{j}\right)}
$$

Clearly, $f_{i}(q) \in(0,1)$ and $\sum_{i} f_{i}(q)=1$, and its partial derivatives are given by

$$
\frac{\partial f_{i}}{\partial q_{j}}(q)=\left\{\begin{array}{cc}
\lambda f_{i}(q)\left(1-f_{i}(q)\right) & \text { for } i=j \\
-\lambda f_{i}(q) f_{j}(q) & \text { for } i \neq j
\end{array}\right.
$$

It follows that

$$
\sum_{j}\left|\frac{\partial f_{i}}{\partial q_{j}}(q)\right|=\lambda f_{i}(q)\left(1-f_{i}(q)\right)+\sum_{j \neq i} \lambda f_{i}(q) f_{j}(q)=2 \lambda f_{i}(q)\left(1-f_{i}(q)\right)
$$


Thus, $\left\|\nabla f^{\prime}\right\|=\max _{i} 2 \lambda f_{i}(q)\left(1-f_{i}(q)\right)$, with the matrix norm defined by $\|P\|=\max _{i} \sum_{j}\left|P_{i j}\right|$ and the corresponding vector norm $\|p\|=\max _{i}\left|p_{i}\right|$. Furthermore,

$$
\frac{\partial^{2} f_{i}}{\partial q_{j} \partial q_{k}}(q)=\left\{\begin{array}{cc}
\lambda^{2} f_{i}(q)\left(1-3 f_{i}(q)+2 f_{i}(q)^{2}\right) & i=j=k \\
\lambda^{2} f_{i}(q) f_{k}(q)\left(2 f_{i}(q)-1\right) & i=j \neq k \\
\lambda^{2} f_{i}(q) f_{j}(q)\left(2 f_{j}(q)-1\right) & i \neq j=k \\
2 \lambda^{2} f_{i}(q) f_{j}(q) f_{k}(q) & i \neq j \neq k
\end{array}\right.
$$

Hence, $\left|\frac{\partial^{2} f_{i}}{\partial q_{j} \partial q_{k}}(q)\right| \leq 2 \lambda^{2}$ for all $i, j, k$. For $i \neq j$, we have

$$
\sum_{k}\left|\frac{\partial^{2} f_{i}}{\partial q_{j} \partial q_{k}}(q)\right| \leq \lambda^{2} f_{i}(q) f_{j}(q)\left(3-2 f_{j}(q)\right) \leq 3 \lambda^{2} f_{i}(q)\left(1-f_{i}(q)\right)
$$

For $i=j$, we have

$$
\sum_{k}\left|\frac{\partial^{2} f_{i}}{\partial q_{j} \partial q_{k}}(q)\right| \leq \lambda^{2} f_{i}(q) f_{j}(q)\left(3-f_{i}(q)\right)
$$

Therefore,

$$
\left\|\nabla^{2} f_{i}\right\| \leq 3 \lambda^{2}
$$

Let $Q$ be a compact subset of the Q-values restricted to the states with a unique optimal decision (in many applications, the structure of the problem ensures that the optimal decision is unique). We can show that the norm of the Jacobian converges to zero uniformly on $Q$. For $n>0$, we have

$$
\lambda^{n} f_{i}(q)\left(1-f_{i}(q)\right) \rightarrow 0 \text { for } \lambda \rightarrow \infty
$$

This convergence follows since, for each $i$, holding $q$ fixed, $\left(1-f_{i}(q)\right) \rightarrow 0$ when $q_{i}=q^{[1]}$ and $f_{i}(q) \rightarrow 0$ when $q_{i}<q^{[1]}$ for $\lambda \rightarrow \infty$. Both converge at an exponential rate and dominate $\lambda^{n}$. Thus, for all $q \in Q$, we have $\left\|\nabla f^{\prime}\right\| \rightarrow 0$ for $\lambda \rightarrow \infty$, implying that $f$ is equicontinuous. Since $Q$ is compact, $f$ is also pointwise bounded, and $f$ converges uniformly on $Q$. We now 
turn to the main result.

\subsection{Convergence}

Theorem: For $q \in Q$ there exists $\lambda^{\star}$ such that $\lambda>\lambda^{\star}$ implies $\left\|q^{(g+1)}-q\right\|$ converges at a geometrical rate. Moreover, the Monte Carlo approximation $\left\|q-q_{M}^{(g+1)}\right\|$ also converges geometrically.

Proof: For $q \in Q$ we assume that $\|q\|$ is bounded. We know that the true $q$ satisfies $q=u+\beta P^{q} q$. Define $q^{(g+1)}=u+\beta P^{q^{(g)}} q^{(g)}$, starting from $q^{(0)}=0$. We can write

$$
q-q^{(g+1)}=\beta P^{q^{(g)}}\left(q-q^{(g)}\right)+\beta\left(P^{q}-P^{q^{(g)}}\right) q
$$

Taking norms and using the fact that $\left\|P^{q^{(g)}}\right\|=1$, we have

$$
\left\|q-q^{(g+1)}\right\| \leq \beta\left\|q-q^{(g)}\right\|+\beta\left\|P^{q}-P^{q^{(g)}}\right\|\|q\|
$$

Now, let $P_{s d}^{q}$ be the $s d$ row of $P^{q}$ and $P_{s_{0} d_{0}, s_{1} d_{1}}^{Q}$ denote the $\left(s_{0} d_{0}\right) \times\left(s_{1} d_{1}\right)$ entry of $P^{Q}$. By Taylor's expansion we have

$$
P_{s_{0} d_{0}, s_{1} d_{1}}^{Q}=P_{s_{0} d_{0}, s_{1} d_{1}}^{q^{(g)}}+\left(q-q^{(g)}\right)^{\prime} \nabla P_{s_{0} d_{0}, s_{1} d_{1}}^{q^{(g)}}+\frac{1}{2}\left(q-q^{(g)}\right)^{\prime} \nabla^{2} P_{s_{0} d_{0}, s_{1} d_{1}}^{q^{\star}}\left(q-q^{(g)}\right)
$$

with $q^{\star}=q^{(g)}+t\left(q-q^{(g)}\right)$ for some $t \in[0,1]$. Hence,

$$
\left\|P^{q}-P^{q^{(g)}}\right\| \leq\left\|\nabla P^{q^{(g)}}\right\|\left\|q-q^{(g)}\right\|+\frac{1}{2}\left\|\nabla^{2} P^{q^{\star}}\right\|\left\|q-q^{(g)}\right\|^{2}
$$

Now we use the properties of the annealing transition kernel that we determined in the 
previous section. At the current $q^{(g)}$, we have

$$
\left\|\nabla P^{q^{(g)}}\right\|=\lambda p_{\lambda}^{q^{(g)}}\left(1-p_{\lambda}^{q^{(g)}}\right)
$$

Moreover, as $p_{\lambda}^{q^{(g)}}$ converges to the (unique) maximum of $q^{(g)}$ with ordered elements $q_{(1)}^{(g)} \leq$ $q_{(2)}^{(g)} \leq \ldots q_{(N-1)}^{(g)}<q_{(N)}^{(g)}$, then as $\lambda \rightarrow \infty$ we have $p_{\lambda}^{q^{(g)}} \rightarrow(0, \ldots, 0,1)$ and $\lambda p_{\lambda}^{q^{(g)}}\left(1-p_{\lambda}^{q^{(g)}}\right) \rightarrow$ 0 as the exponential term is dominated by $e^{\lambda\left(q_{(N)}^{(g)}-q_{(N-1)}^{(g)}\right)}$.

Hence, we can choose $\lambda^{\star}$ such that $\forall \lambda^{(g)} \geq \lambda^{\star}$ we have

$$
\left\|\nabla\left(P^{q^{(g)}}\right)\right\|\|q\|<\frac{1-\beta}{2}
$$

Therefore, for $\lambda^{(g)} \geq \lambda^{\star}$, we have that

$$
\left\|q-q^{(g+1)}\right\| \leq L\left\|q-q^{(g)}\right\|+\frac{\beta}{2}\left\|\nabla^{2} P^{q^{\star}}\right\|\|q\|\left\|q-q^{(g)}\right\|^{2}
$$

where $L=(1+\beta) / 2<1$, using the Taylor expansion for $\left\|P^{q}-P^{q^{(g)}}\right\|$ and the inequality for $\left\|q-q^{(g+1)}\right\|$.

Under the properties of the annealing function

$$
\left\|\nabla^{2} P^{q^{\star}}\right\| \leq 3\left(\lambda^{(g)}\right)^{2} \leq 3\left(\lambda^{\star}\right)^{2}
$$

Moreover, from the boundedness assumption on $\|q\|$, we have $\frac{1}{2} \beta\left\|\nabla^{2} P^{q^{\star}}\right\|\|q\| \leq K$ for some finite constant $K<\infty$. From (1), we can write

$$
\left\|q-q^{(g+1)}\right\| \leq L\left\|q-q^{(g)}\right\|+K\left\|q-q^{(g)}\right\|^{2}
$$


Let $d_{g+1}=\left\|q-q^{(g+1)}\right\|$ so that $\forall \lambda^{(g)} \geq \lambda^{\star}$ we have

$$
d_{g+1} \leq L d_{g}+K d_{g}^{2}, \text { where } 0<L<1, K<\infty
$$

We now need to show that the sequence $d_{g}$ has geometric convergence. To do this, define the sequence $a_{g}$ by $a_{g+1}=L a_{g}+K a_{g}^{2}$ and note that this has the property that $d_{g} \leq a_{g}$ implies $d_{g+1} \leq a_{g+1}$. Hence, if we can show that the sequence $a_{g}$ converges geometrically, this will imply $d_{g+1}$ converges geometrically.

As $0<L<1$, we can define $c=(1-L) / 2 L$. Consider first the case when $a_{g}>c L / K$, or equivalently $L \leq K a_{g} / c$. Then

$$
a_{g+1}=L a_{g}+K a_{g}^{2} \leq\left(1+\frac{1}{c}\right) K a_{g}^{2}=\frac{1+L}{1-L} K a_{g}^{2}
$$

and there exists $\xi$ such that $a_{g} \leq C \xi^{g}$ and there is geometric convergence.

Secondly, in the case when $a_{g} \leq c L / K=(1-L) / 2 K$, which happens for large $g$, we have

$$
a_{g+1} \leq(1+c) L a_{g}=\frac{1+L}{2} a_{g}
$$

and as $(1+L) / 2<1$, the sequence $a_{g}$ converges geometrically. In turn, this implies that $d_{g}$ converges geometrically, as required.

Finally, we have to show that the Monte Carlo error in the approximation is not too large. Specifically, we show that $\left\|q-q_{M}^{(g+1)}\right\| \leq L_{M}\left\|q-q^{(g)}\right\|$ with $L_{M}<1$ for a suitably large $M$. We have the random mapping $q_{M}^{(g+1)}=u+\beta \mathcal{P}_{M}^{q^{(g)}} q_{M}^{(g)}$. We now write $q_{M}^{(g+1)}=q^{(g+1)}+\epsilon_{M}^{(g+1)}$ conditional on $q^{(g)}$ to get the norm inequality

$$
\left\|q-q_{M}^{(g+1)}\right\| \leq\left\|q-q^{(g+1)}\right\|+\left\|\epsilon_{M}^{(g+1)}\right\|
$$


If we pick $M$ large enough so that $\left\|\epsilon_{M}^{(g+1)}\right\| \leq \epsilon_{M}\left\|q-q^{(g+1)}\right\|$ where $\epsilon_{M}=(1-L) / 2$, say, then again we have first order convergence. When the error is sufficiently small $\left\|q-q^{(g+1)}\right\| \leq \frac{\epsilon L}{K}$ and therefore

$$
\left\|q-q_{M}^{(g+1)}\right\| \leq(1+\epsilon) L_{M}\left\|q-q_{M}^{(g)}\right\|
$$

where $L_{M}=L+\epsilon_{M}<1$, as required.

The variance estimate can be determined as follows. For the whole vector, we can argue that we have a random variable $\hat{q}^{(g)}=\frac{1}{M} \sum_{m=1}^{M} \hat{q}_{m}^{(g)}$ as a Monte Carlo estimator has the property $E\left(\hat{q}^{(g)}\right)=q^{(g)}$. By definition

$$
\left\|\hat{q}_{M}^{(g)}-q^{(g)}\right\|=\sup _{(s, d)}\left|\frac{1}{M} \sum_{m=1}^{M} q_{m, s d}^{(g)}-Q_{s d}^{(g)}\right|
$$

and by Chebyshev's inequality we have

$$
P\left(\left|\hat{q}_{M}^{(g)}-q^{(g)}\right| \geq \epsilon\left\|u^{(g)}\right\|\right) \leq \frac{\operatorname{Var}\left(\hat{q}_{M}^{(g)}\right)}{M \epsilon^{2}\left\|u^{(g)}\right\|^{2}}
$$

Now, we have the inequalities

$$
\left|\hat{q}_{m}^{(g)}\right| \leq\left\|u^{(g)}\right\| /(1-\beta) \text { and } \operatorname{Var}\left(\hat{q}_{M}^{(g)}\right) \leq\left\|u^{(g)}\right\|^{2} /(1-\beta)^{2}
$$

Therefore, we have the bound

$$
P\left(\left|\hat{q}_{M}^{(g)}-q^{(g)}\right| \geq \epsilon\left\|u^{(g)}\right\|\right) \leq \frac{1}{M \epsilon^{2}(1-\beta)^{2}}
$$

Hence, we have a geometric convergence result. The Monte Carlo estimate can be computed in $O(N)$. Notice that we need to increase $M$ the closer we get to the solution as, otherwise, Monte Carlo errors dominate and the solution may oscillate. 
Contrast this with the standard $Q$-Learning convergence (see, Watkins and Dayan, 1992).

The latter depends on a stochastic approximation argument, see Robbins and Munro (1951) and Chen and White (2002). Convergence requires the specification of a suitable sequence of learning coefficients with the rate being sensitive to this choice, and there is no geometric convergence available.

\section{Application: Stochastic Investment Problem}

To illustrate our methodology, consider a dynamic stochastic investment and consumption problem, see Judd (1999) and Ljungqvist and Sargent (2004) for further applications. Suppose that an agent is exposed to a random but persistent labor shock and faces a choice between consumption and saving. The agent's problem is to maximize the future discounted expected utility of consumption.

The general Bellman equation is

$$
V(s)=\max _{d}\left(u(s, d)+\beta \int V\left(s^{\prime}\right) p\left(s^{\prime} \mid s, d\right) d s^{\prime}\right)
$$

More specifically, the value function is

$$
V(s)=\max _{\left\{c_{t}\right\}} E\left(\sum_{t=0}^{\infty} \beta^{t} u\left(c_{t}\right)\right)
$$

where utility preferences $u\left(c_{t}\right)$ over consumption $c_{t}$ are represented by the standard constant relative risk aversion (CRRA) utility.

$$
u_{t}\left(c_{t}\right)=\frac{c_{t}^{1-\gamma}}{1-\gamma}
$$

and $\gamma$ is a given risk aversion parameter. 
We study two cases. First, assume that the agent can only save by investing in a risky security, say stocks. The state space is $s_{t}=\left(w_{t}, l_{t}\right)$ consisting of the agent's current wealth and labor. The decision space is one-dimensional, consisting of the agent's consumption choice $d_{t}=c_{t}$. Second, we assume that the agent faces an additional portfolio decision and must chose between investing in a safe and a risky security. The safe asset earns a riskfree return denoted $r_{f}$. This will lead to a two dimensional decision variable $d_{t}=\left(c_{t}, b_{t}\right)$, including the amount allocated to the safe investment.

In the first case, the wealth evolves according to the transition equation

$$
w_{t+1}=\left(w_{t}+l_{t}-c_{t}\right) \exp \left(R_{e}\right)
$$

where $\exp \left(R_{e}\right)$ is the risky return, following a log-normal distribution, defined by

$$
R_{e} \sim \mathcal{N}\left(\mu_{e}, \sigma_{e}^{2}\right)
$$

The agent's labor income is $l_{t}$, which develops with transition probability

$$
l_{t+1}=l_{t} \exp \left(R_{l}\right) \text { with } R_{l} \sim \mathcal{N}\left(\mu_{l}, \sigma_{l}^{2}\right)
$$

The $Q$-values are then defined by

$$
\begin{aligned}
Q(s, d) & =u(s, d)+\beta \int V\left(s^{\prime}\right) p\left(s^{\prime} \mid s, d\right) d s^{\prime} \\
& =\frac{c_{t}^{1-\gamma}}{1-\gamma}+\beta \int \max _{d} Q\left(s^{\prime}, d\right) p\left(s^{\prime} \mid s, d\right) d s^{\prime}
\end{aligned}
$$




\subsection{Case I: Risky Asset Only}

The state $s_{t}=\left(l_{t}, w_{t}\right)$ contains $l_{t}$ the current labor income and $w_{t}$ the wealth at the beginning of the period. Then labor and consumption evolve according to the transition rule

$$
\begin{aligned}
l_{t+1} & =l_{t} \exp \left(R_{l}\right) \\
w_{t+1} & =\left(w_{t}+l_{t}-c_{t}\right) \exp \left(R_{e}\right)
\end{aligned}
$$

The agent's decision variable is to choose the amount consumed. This is equivalent to choosing the amount saved, since $d_{t}=\left(w_{t}+l_{t}-c_{t}\right)$, and we constrain the saving to be non-negative. We now study a number of different model specifications of this model.

Figure 1 shows the value function for a range $\gamma \in\{3.5,2.7,0.8,0.2,0\}$ from top to bottom. We use a discount rate $\beta=0.8$. A Monte Carlo sample size of $M=500$ leds to good convergence. The black line corresponds to the state with wage $=1$ and the blue for wage $=0$ in the same wealth state $s_{1}$. The state transition probabilities are $p\left(s^{\prime} \mid s, d\right) \sim$ $\mathcal{L N}\left(0.04,0.1^{2}\right)$ corresponding to $\mu=\sigma=0.1$. This leads to a positive net present value given by the condition

$$
\beta E\left[e^{R}\right]=\beta e^{\mu+\frac{\sigma^{2}}{2}}=0.84<1
$$

Even though the parameter $\gamma$ expresses an agent's attitude to risk in the static case the optimal behavior is different in the dynamic context. Bellman's equation implies that total utility is the sum of separably evaluated utilities at each time with the value of each state is the sum of present utility and present value of expected future utility. Hence, an agent with large value of $\gamma$ invests more than agent with small $\gamma$ as consuming a small amount of money many times gives more cumulative utility than consuming a large amount at one time. Conversely, an agent with $\gamma=0$ is risk neutral with linear utility function and is only concerned about the total net present value. The different slopes in Figure 1 and 2 arises 
from the dynamic nature of the problem and the specification of $(\beta, \mu, \sigma)$ and not the agents' attitude towards risk.

Figure 2 shows the corresponding policy function corresponding to the optimal level of investment. For large $\gamma$, e.g. $\gamma \geq 3.5$, the optimal decision is a straight line. In the region $3.5 \geq \gamma \geq 0.8$, the decision is a little curved flattening out at the end of the decision region due to a truncation effect. Finally, for $0.8 \geq \gamma$, the optimal policy is a straight line with slope less than one. The optimal investment is far enough from the boundary not to be affected by truncation effects. The truncation effect increases when $\mu$ or $\sigma$ are not too large and smaller $\sigma$ values tend to lead to larger MC errors.

Figure 3 illustrates a case where the expected net present value of investment is negative, corresponding to a discount rate of $\beta=0.95$ with state transition probabilities $p\left(s^{\prime} \mid s, d\right) \sim$ $\mathcal{L N}\left(0.1,0.1^{2}\right)$ and a Monte Carlo sample size $M=500$. Here, the present value condition becomes

$$
\beta E\left[e^{R}\right]=\beta e^{\mu+\frac{\sigma^{2}}{2}}=1.06>1
$$

There is a difference in the optimal investment decision. This leads agent to invest all and consume nothing as would a risk neutral agent in this case. The only difference in Figure 3 is a truncation effect that varies with $\gamma$ near boundary.

\subsection{Case II: Risky and Safe Assets}

We now extend the above problem to allow the agent to invest in both a risk-free bond and the risky equity security. The risk-free bond earns a rate of $r_{f}$. The decision is now $d_{t}=\left(e_{t}, b_{t}\right)$. The state space is still $s_{t}=\left(l_{t}, w_{t}\right)$, but the evolution of the state is now 
governed by the transition rule

$$
\begin{aligned}
l_{t+1} & =l_{t} \exp \left(R_{l}\right) \\
w_{t+1} & =e_{t} \exp \left(R_{e}\right)+b_{t} \exp \left(r_{f}\right)
\end{aligned}
$$

Consumption is now given as $c_{t}=w_{t}+l_{t}-s_{t}-b_{t}$. Both $s_{t}$ and $b_{t}$ are bounded to be nonnegative. We choose a bounded state space $s_{t} \in[0,10] \times[0,10]$. This leads to a truncation effect for the transition probabilities.

The total optimal investment looks good in the region $w_{t}+l_{t}<10$. However, the proportion of investment in stock and bond is severely distorted in the upper triangle region of the state space $w_{t}+l_{t}>10$ due to truncation of the transition probability. Since money from stock investment cannot exceed upper limit of wealth region [0,10], actual stock return inevitably has a negative mean by using truncated probability in that region even though we set $\mu=0.1$. Therefore, investment in the bond is abnormally preferred in that region.

One could extend the range of the state space to fix the truncation problem, but if the value function is close to plane there is no incentive to balance the investment in stock and bond in our current model. Hence, the optimal investment decision between stock and bond could be unstable: either all in stock or all in bond. This can also add to large MC errors.

Since the discount factor $\beta$ is constant, this model has difficulty in explaining the equity premium of historical returns. This can be seen from the observation that in a region of interest rates between $2-5 \%$, the stock investment is absolutely preferred to bond in the lower triangle region of state space $w_{t}+l_{t}<10$. To obtain an equity premium for low values of $\gamma$ or $r_{f}$ would require a recursive utility model.

The arguments about risk aversion in the univariate case also apply here. One avenue for further exploration is the risk-sensitive operator approach of Hansen and Sargent (1995). This would help in providing smoothly balanced optimal investment portfolios of stock and 
bond rather than extremal solutions.

\section{Conclusion}

In this paper we propose a new class of Monte Carlo algorithms for computing the $Q$-values of dynamic programming and reinforcement learning. We provide a geometric convergence bound. The algorithm is scalable in being $O(N)$ in computation. The main advantages are implementability in both discrete and continuous state and decision spaces together with scalability. By using sequential Monte Carlo to evaluate the random operator $\mathcal{P}^{(g)}(s, d)$ we can simplify the problem and calculate the $Q(s, d)$ values at any point in the state-decision space. The algorithm performs well on a stochastic investment problem in multi-dimensions. There are a number of possible directions for future research. First, the sampling distribution used to construct the random operator could depend on $Q^{(g)}$ or on $p\left(s^{\prime} \mid s, d\right)$. For example, an efficient chocie might be to use the current residual to provide a data-adaptive choice of the grid density $p^{(g)}\left(s^{\prime}, d^{\star}\right)$. We do not explore this further here. Second, applications to higher dimensional decision spaces are challenging.

\section{References}

Aarts, E and Korst, J. (1989). Simulated Annealing and Boltzmann Machines. Wiley, New York.

Amzal, B., Bois, F.Y., Parent, E. and C.P. Robert (2006). Bayesian-Optimal Design via Interacting Particle Systems Journal of American Statistical Association, 101, 773-785.

Barto, A. and M. Duff (1994). Monte Carlo Matrix Inversion and Reinforcement Learning. In: Advances in Neural Information Processing Systems, 6, 687-694.

Bellman, R. (1957). Dynamic Programming. Princeton University Press. 
Bellman, R., Kalaba, R. and B. Kotkin (1963) Polynomial approximation-A new computational technique in Dynamic programming: allocation processes. Mathematics of Computation, $17,155-161$.

Bertsekas, D. (1994) Dynamic Programming and Optimal Control (vols I and II). Athena Scientific, Belmont, MA.

Bertsekas, D. and Tsitsiklis, J.N. (1996) Neuro-Dynamic Programming. Athena Scientific, Belmont, MA.

Brockwell, A.E. and Kadane, J.B. (2003). A gridding method for Bayesian sequential decision problems. Journal of Computational and Graphical Statistics, 12(3), 566-584.

Brown, P.B., J. Smith and P. Sun (2010). Information relaxations and duality in stochastic dynamic programs. Operations Research, 58(4), 785-801.

Casella, G. and Robert, C. (2005). Monte Carlo Statistical Methods. Springer-Verlag, NY.

Chen, X. and White, H. (2002). Asymptotic properties of some projection-based RobbinsMunro procedures in a Hilbert space. Studies in Nonlinear Dynamics and Econometrics, $6(1), 1-55$.

Curtiss, J. (1954). A Theoretical Comparison of the Efficiencies of Two Classical methods and a Monte Carlo Method for Computing One Component of the Solution of a set of Algebraic Equations. In Symposium on Monte Carlo Methods (ed H. A. Mayer), 191-233. Wiley: New York.

Cutkosky, R.E. (1951). A Monte Carlo method for Solving a Class of Integral Equations. J. Res. Nat. Bur. Stand., 47, 113-115.

Dayan, P. (1992). The Convergence of $T D(\lambda)$ for general $\lambda$. Machine Learning, 8, 341-362. 
Diaconis, P. and David Freedman (1999). Iterated Random Functions. SIAM Review, 41(1), 45-76.

Dunford, N. and Schwartz, J.T. (1958). Linear Operators: Part I, General Theory New York: Interscience.

Forsythe, G.A. and Leibler, R.A. (1950) Matrix Inversion by a Monte Carlo Method. Math. Tables and Computation, 4(31), 127-9.

Gittins, J.C. (1979). Bandit processes and dynamic allocation indicies (with discussion). Journal of Royal Statistical Society B, 41, 148-77.

Halton, J.H. (1970). A retrospective and propective survey of the Monte Carlo method. SIAM Review, 12(1), 1-63.

Halton, J.H. (2006). Sequential Monte Carlo techniques for solving non-linear systems. Monte Carlo Methods and Applications, 12(2), 113-141.

Hammersley, J.M. and Handscomb, D.C. (1964) Monte Carlo Methods. Chapman and Hall. Howard, R. (1960). Dynamic Programming and Markov Processes. MIT Press, Cambridge. Jakkola, T., Jordan, M. I. and Singh, S.P. (1994). On the Convergence of Stochastic Iterative Dynamic Programming Algorithms. Neural Computation, 6, 1185-1201.

Judd, K. (1998). Numerical Methods in Economics. MIT Press, Cambridge, MA.

Judd, K. and A. Solnick (1994). Numerical Dynamic Programming with Shape-Preserving Splines. Working Paper, Hoover Institution.

Kearns, M. and S. Singh (1998). Near-Optimal Reinforcement Learning and Polynomial Time. Proc 15th Conference on Machine Learning, 260-268. 
Kearns, M. and S. Singh (1999). Finite-Sample Convergence rates for Q-Learning and Indirect Algorithms. In Advances in Neural Information Processing, 996-1002.

Kirkpatrick, S. (1984). Optimization by simulated annealing: quantitative studies. Journal of Statistical Physics 34, 975-986.

Kirkpatrick, S., C.D. Gelatt and M.P. Vecchi (1983). Optimization by simulated annealing. Science 220, 671-680.

Kushner, H. J. and D. S. Clark (1978). Stochastic Approximation Methods for Constrained and Unconstrained systems, Springer-Verlag, NY.

Lagondakis, M.G. and R.E. Parr (2003). Least-Squares Policy Iteration. Journal of Machine Learning Research, 1107-1149.

Lagondakis, M.G., R.E. Parr and M.L.Littman (2002). Least-Squares Methods in Reinforcement learning for Control. Lecture Notes on AI, Springer, 249-260.

Ljungquist, L. and T. Sargent (2004). Recursive Macroeconomic Theory. MIT Press.

Lovejoy, W.S. (1991). A Survey of Algorithmic Methods for Partially Observed Markov Processes Annals of Oper. Res., 18, 47-66.

Mueller, P. (2000). Simulation-based Sequential Design. in Bayesian Statistics 6, (eds Bernardo et al), 459-474. Oxford University Press.

Mueller, P., Sanso, B. and M. De Iorio (2004). Optimal Bayesian Design by inhomogeneous Markov chain simulation. Journal of American Statistical Association, 99, 788-798.

Page, E.S. (1954). The Monte Carlo Solution of some Integral Equations. Proc. Camb. Phil. Soc, 58, 57-78. 
Polson, N.G. and G.O. Roberts (1993). Discussion: From Image Deblurring to Optimal Investments: Maximum Likelihood solutions for Positive Inverse Problems. Journal of Royal Statistical Society B, 607-608.

Pincus, M. (1968). A Closed Form Solution of Certain Dynamic Programming Problems. Oper. Research., 18, 1225-1228.

Puterman (1994). Markov Decision Processes. Wiley: New York.

Robbins, H. and Munro, S. (1951). A Stochastic Approximation Method. Annals of Statistics, 22, 400-407.

Rudin, W. (1976). Principles of Mathematical Analysis. McGraw-Hill.

Rust, J. (1997). Using Randomization to Break the Curse of Dimensionality Econometrica, $65,3,487-516$.

Rust, J. (2000) Structural Estimation of Markov Decision Processes. Handbook of Econometrics, 4, 3082-3139.

Sargent, T. and Ljungquist, L. (2004). Recursive Macroeconomic Theory. MIT Press.

Singh, S., Jakkola, T., Littman, M.L., C. Szepesvari (2000). Convergence Results for singlestep on-policy reinforcement learning algorithms. Machine Learning, 38(3), 287-308.

Sutton, R. and Barto, A.G. (1998). Reinforcement Learning. MIT Press, Cambridge, MA.

Sutton, R. (1999). Open theoretical questions in Reinforcement Learning. Procs. EuroCOLT99, 11-17.

Tauchen, G. (1990) Solving the Stochastic Growth Model by Using Quadrature Methods and Value-Function Iteration. Journal of Business and Economic Statistics, 8, 49-51. 
Tauchen, G. and Hussey, R. (1991) Quadrature-Based Methods for Obtaining Approximate Solutions to Nonlinear Asset Pricing Models. Econometrica, 59, 371-196.

Tesauro, G. and Galperin, G.R. (1997). On-line policy improvement using Monte Carlo search. Advances in Neural Information Processing, 1068-1074.

Tsitsiklis, J.N. (1994). Asynchronous Stochastic Approximation and Q-Learning. Machine Learning, 16, 185-202.

Tsitsiklis, J.N. (2002). On the Convergence of Optimistic Policy Iteration. Journal of Machine Learning Reserach, 3, 59-72.

Metropolis, N. and Ulam, S. (1949) The Monte Carlo method. Journal of American Statistical Association, 44, 335.

Virto, M., Martin, J, Rios Insua, D. and Moreno Diaz, A. (2003). Approximate solutions to semi-markov decision processes through MCMC methods. In Eurocast 2003, 151-162.

Watkins, C.J.C.H. (1989). Learning from Delayed Rewards. PhD Thesis, Cambridge University, England

Watkins, C.J. and Dayan, P. (1992). Q-Learning. Machine Learning, 8, 279-292.

Whittle, P. (1969). A view of Stochastic Control theory. Journal of Royal Statistical Society A, $132(3), 320-324$.

Whittle, P. (1983). Optimization over Time: Vols I and II. Wiley: New York.

Whittle, P. and Komarova, N. (1988). Policy Improvement and the Newton-Rapheson Algorithm. Prob. in Eng. and Info. Sciences, 2, 249-255.

Whitt, W. (1978) Approximations of Dynamic Programs I. Math Operations Research, 3, 231-243. 

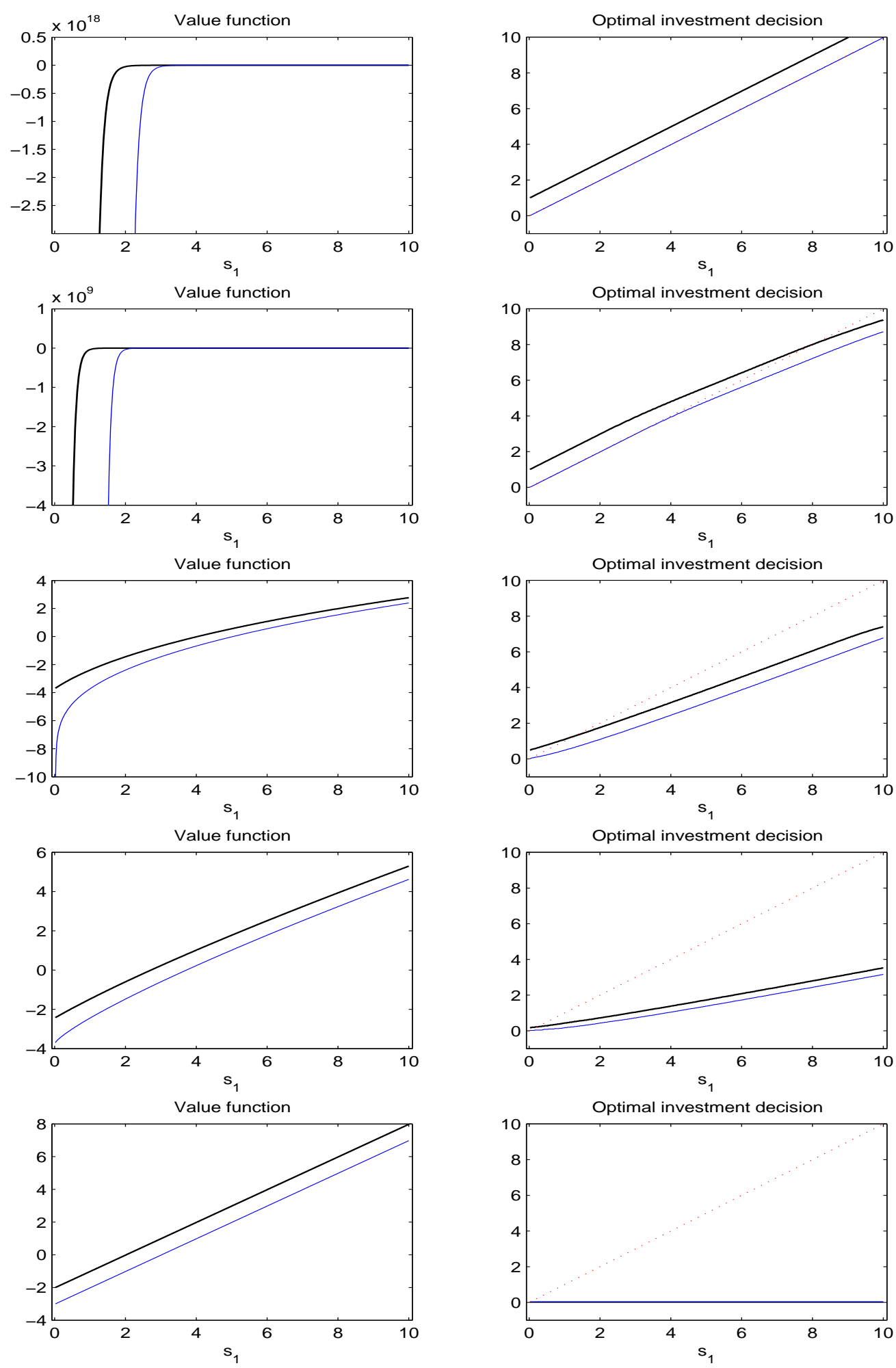

Figure 1: Value function and optimal investment decision. The values of $\gamma$ used in subplots are $(3.5,2.7,0.8,0.2,0)$ from top to bottom. The black line corresponds to the state with wage $=1$ and the blue for wage $=0$ in the same wealth state $s_{1}$. The model specification and parameters correspond to a discount rate of $\beta=0.8$ with state transition probability $p\left(s^{\prime} \mid s, d\right) \sim \mathcal{L N}\left(0.04,0.1^{2}\right)$ corresponding to $\mu=0.1, \sigma=0.1$. The Monte Carlo sample size is $M=500$. 


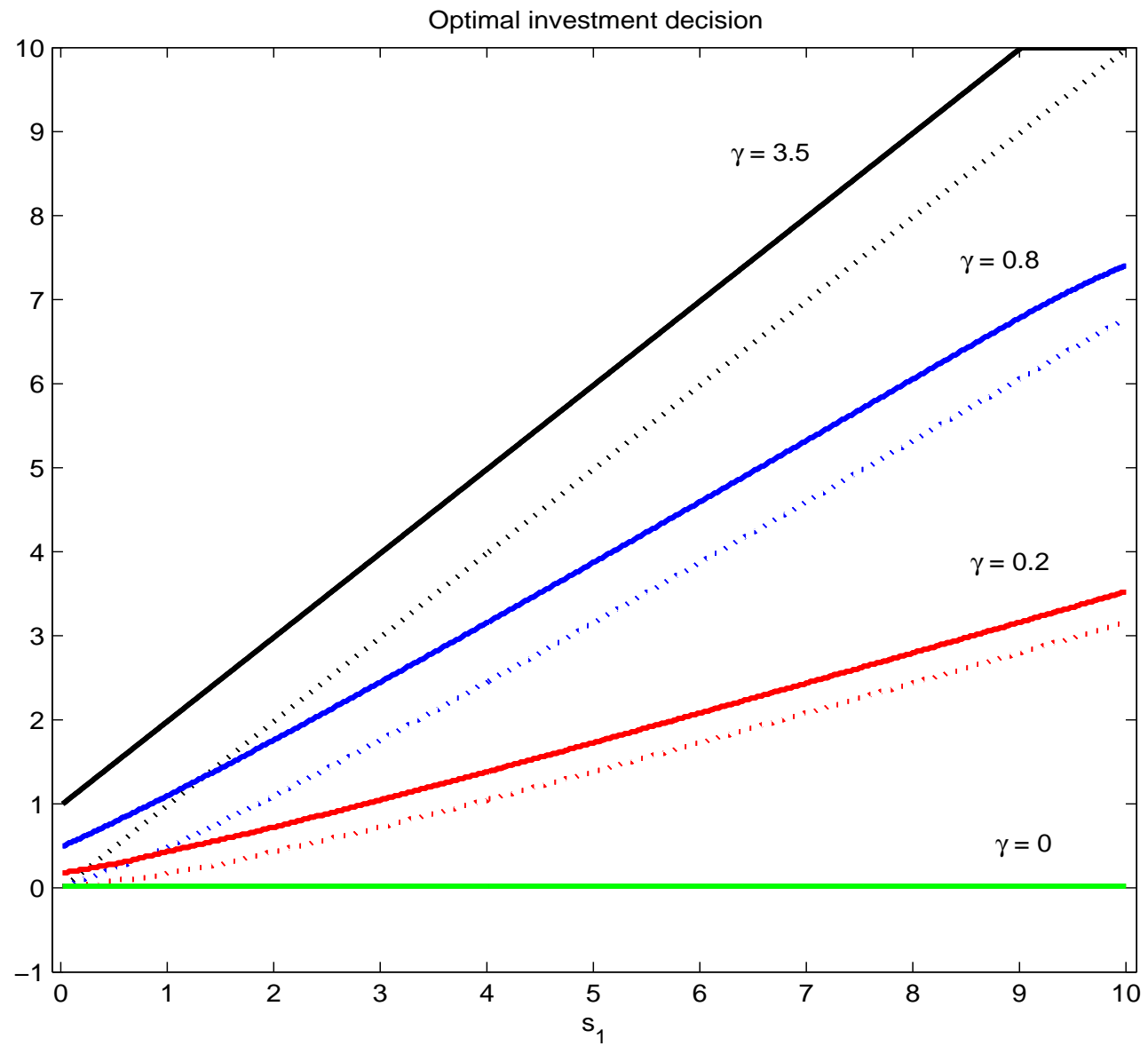

Figure 2: Optimal investment decision in the case of positive net present value where $\beta E\left[e^{R}\right]=\beta e^{\mu+\frac{\sigma^{2}}{2}}<1$. Solid line stands for state with wage $=1$ and dotted line for wage $=0$ in the same wealth state $s_{1}$. Model settings as in Figure 1. 

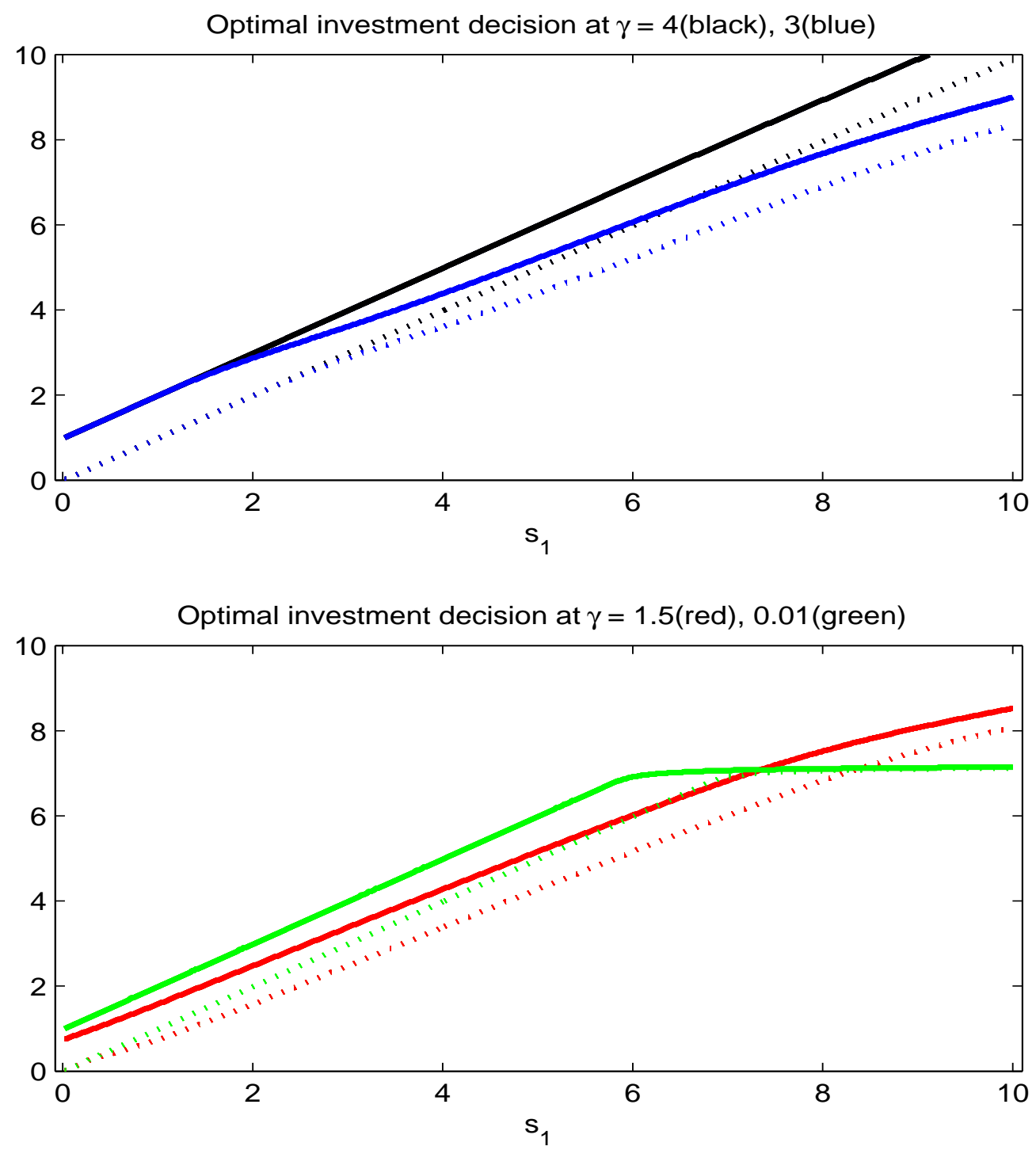

Figure 3: Optimal investment decision in the case of negative net present value with $\beta E\left[e^{R}\right]=$ $\beta e^{\mu+\frac{\sigma^{2}}{2}}>1$. Solid line stands for state with wage $=1$ and dotted line for wage $=0$ in the same wealth state $s_{1}$. Here we use a discount rate $\beta=0.95$ with state transition probability $p\left(s^{\prime} \mid s, d\right) \sim \mathcal{L N}\left(0.1,0.1^{2}\right)$ and a Monte Carlo sample size $M=500$. 

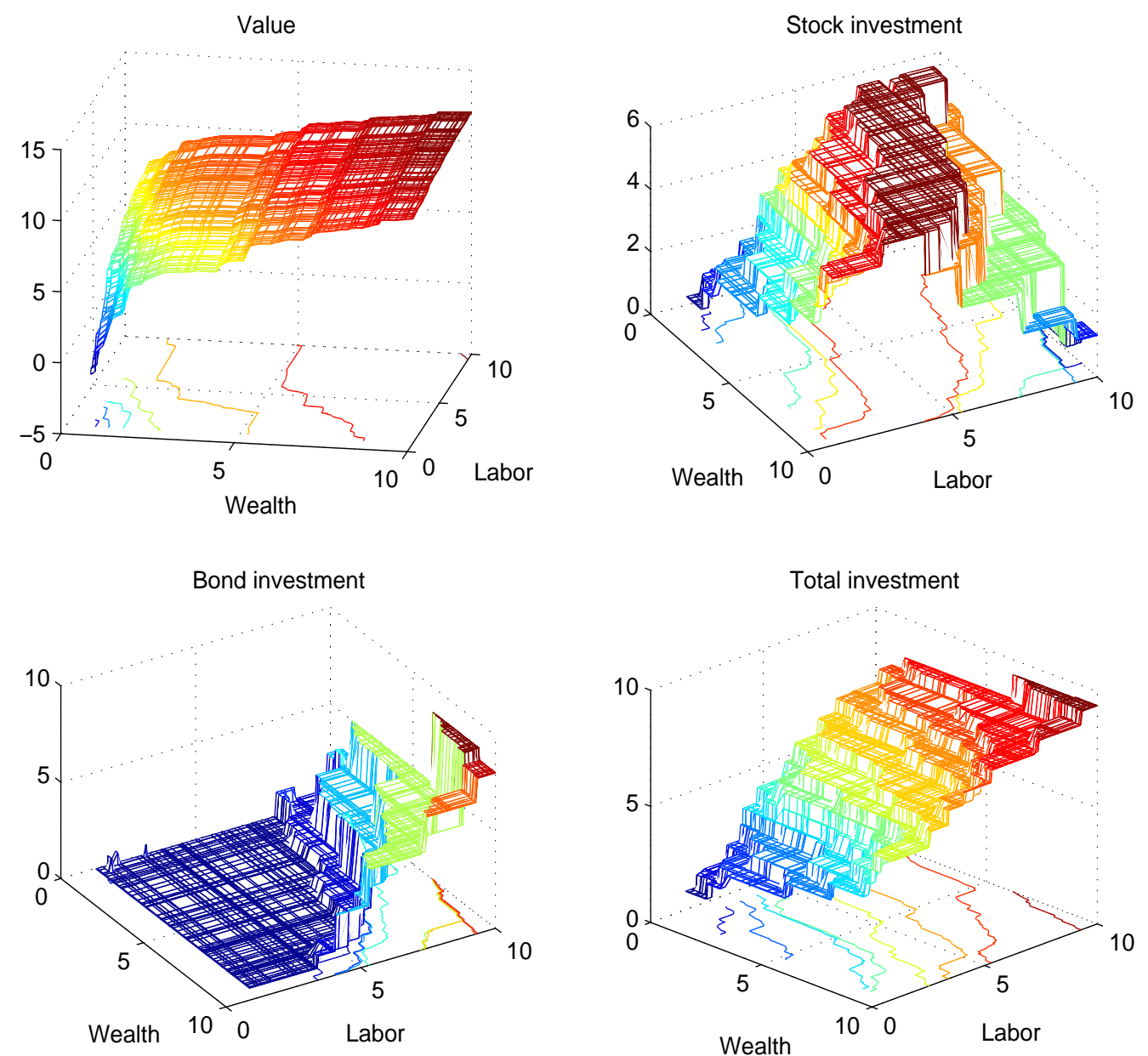

Figure 4: Stochastic investment in 2-dimensional case with continuous $s(100 \times 100)$ discrete $d(15 \times 15)$ where $r_{f}=0.02$ and logarithmic $(\gamma=1)$ utility. 

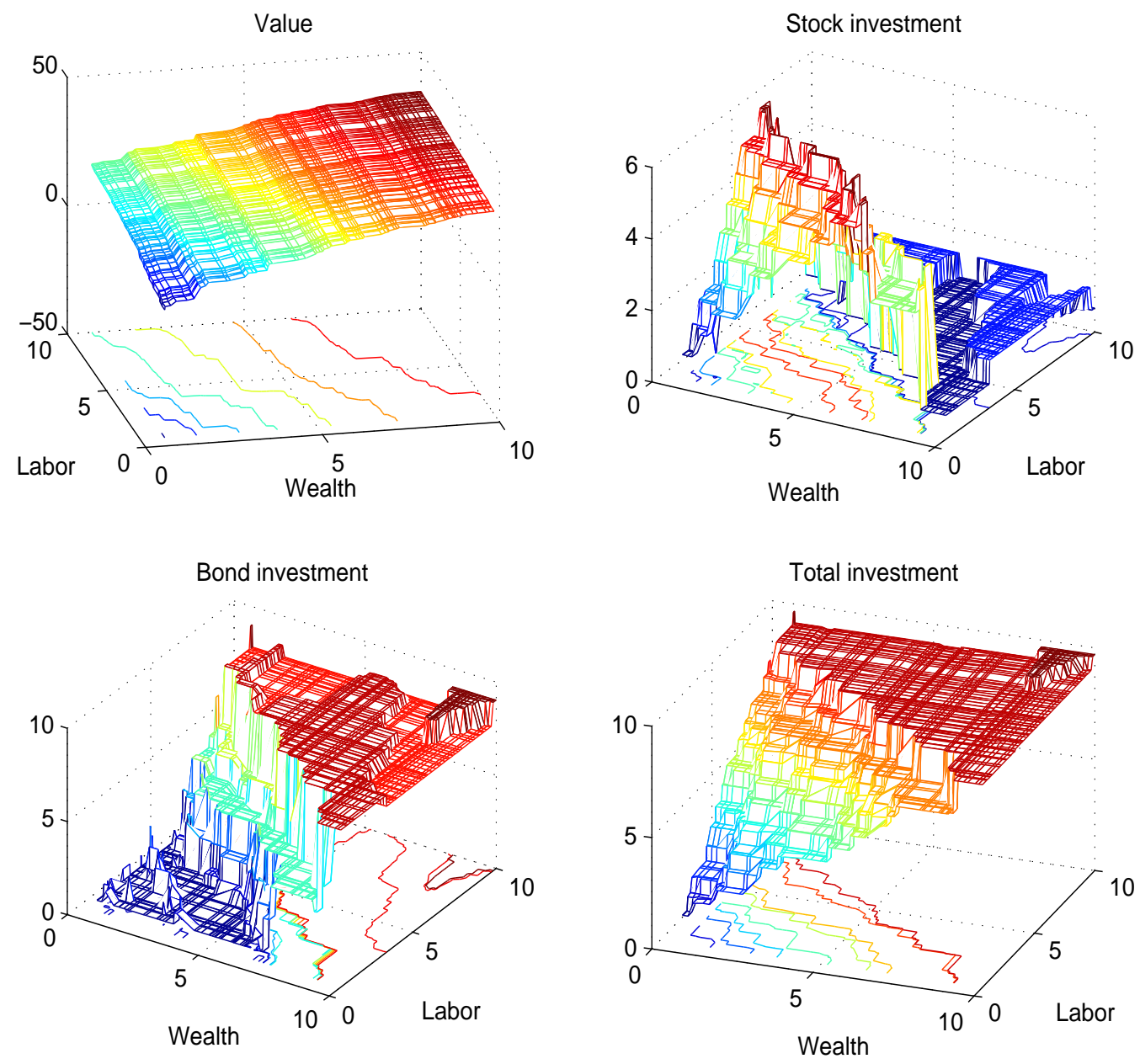

Figure 5: Stochastic investment in 2-dimensional case with continuous $s(70 \times 70)$ discrete $d(15 \times 15)$ where $r_{f}=0.05$ risk aversion $\gamma=0.3$ 